\title{
Analytical band centrifugation revisited
}

\author{
Cornelia M. Schneider ${ }^{1} \cdot$ Helmut Cölfen $^{1}[$
}

\begin{abstract}
Analytical band centrifugation (ABC) is a powerful tool for the analysis of macromolecules and nanoparticles. Although it offers several advantages over the sedimentation velocity (SV) experiment like a physical separation of the individual components and the possibility to perform chemical reactions, its analysis is still very restricted. Therefore, we investigated the integration of $\mathrm{ABC}$ data as an alternative approach, as this results in data similar to SV, which can then be evaluated by many established evaluation programs. We investigated this method using two different test systems, myoglobin as a biopolymer with significant diffusion and $100 \mathrm{~nm}$ polystyrene latex as a large particle with negligible diffusion, and found some limiting issues. These are namely, broadening of the initial boundary by diffusion of the sample, which can be taken into account and the dynamic density gradient between the solvent in the sector and the overlaid solution, which deforms the initial band upon movement through the gradient and is currently not taken into account. We show the influence these two factors have on the evaluation and show that it is possible to calculate the time-dependent change in solvent density and viscosity in the AUC cell using the integrated form of Fick's second law. We conclude that taking the dynamic density gradient into account will open $\mathrm{ABC}$ for the sophisticated methods based on the analysis of the whole sedimentation boundary and not just the determination of an average sedimentation coefficient.
\end{abstract}

Keywords Analytical band centrifugation · Analytical ultracentrifugation · Diffusion · Dynamic density gradient

\section{Introduction}

Analytical band centrifugation $(\mathrm{ABC})$ is a variation of the sedimentation velocity (SV) experiment in the analytical ultracentrifuge (AUC). It was performed for the first time by Vinograd et al. (1963). In this experiment, one solution can be held separated from the solution in the sample sector in a small reservoir, which is connected to the sector via thin capillaries. Upon acceleration of the rotor, the solution is overlaid on top of the other, as also shown in the overview in Fig. 1 on the left. Vinograd did a lot of pioneer work on this type of experiments, as he tested different centerpieces and

Helmut Cölfen

Helmut.Coelfen@uni-konstanz.de

1 Physical Chemistry, University of Konstanz, Universitätsstrasse 10, 78457 Constance, Germany their advantages/disadvantages (Vinograd et al. 1965) and investigated the mechanism and conditions of this experiment (Vinograd and Bruner 1966a, b).

In a standard SV AUC experiment, a sample solution or dispersion is filled into the sample sector and distributed evenly in the sector. The sample starts to sediment down towards the bottom of the cell by the centrifugal force created by the spinning rotor. The formed concentration profile is recorded with a detector, which can be an UV/Vis detector, interference, fluorescence or Schlieren detector. Evaluation of the concentration profile delivers parameters of the sample under investigation like sedimentation coefficient distribution, diffusion coefficient distribution, and, applying further knowledge of the sample, molar mass distribution, density and frictional coefficient distribution (Mächtle and Börger 2006). For particles, AUC provides a broad application range from below $1 \mathrm{~nm}$ up to $5 \mu \mathrm{m}$ and a very high resolution in the Ångström range (Cölfen and Pauck 1997; Mächtle and Börger 2006). In an ABC experiment, the sample is not filled into the sample sector, but in concentrated form and small volumes into the reservoir. For strong non-ideal samples, this high concentration 
Analytical Band Centrifugation

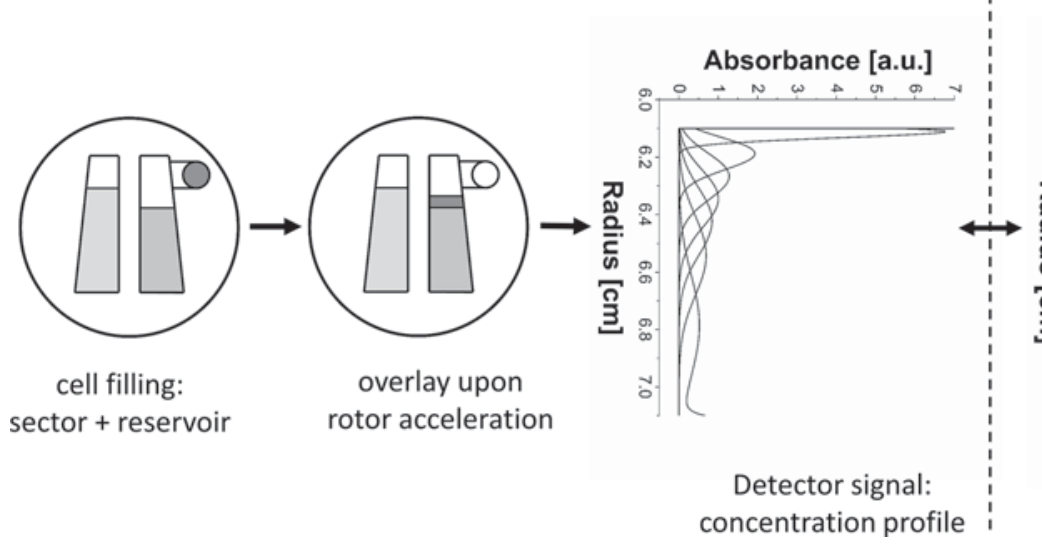

AUC sedimentation velocity experiment

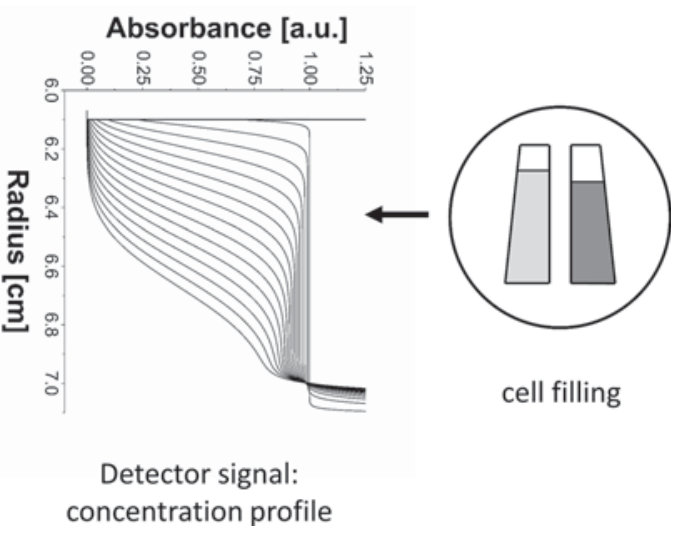

Fig. 1 Scheme of the principle of $\mathrm{ABC}$ in comparison to the SV experiment. Shown are the sample fillings and the resulting sedimentation concentration profiles. For the $\mathrm{ABC}$ experiment, the sample is filled in the reservoir and is overlaid onto the solvent solution with a slightly higher density in the sample sector during rotor acceleration. This results in Gaussian shaped concentration profiles for a monodisperse sample in comparison to the sigmoidal shaped concentration profiles in the SV experiment may cause problems in ABC experiments. Upon overlay, a sharp boundary is formed by providing a small density difference in between the solutions with a slightly higher density of the solvent as compared to the overlaid solution to avoid convection problems and to create the needed sharp band of the sample. Therefore, for a monodisperse sample, a Gaussian shape concentration profile is formed instead of a sigmoidal shaped profile as in the SV experiment, which is also graphed in Fig. 1 in the middle. This difference offers some opportunities, which are shown in the following examples.

The first ABC experiment was conducted by Vinograd et al. (1963). They presented this method as a different approach for the characterization of samples in comparison to the known SV method. In the experiment, a solution of macromolecules of a volume between 25 and $50 \mu$ was overlaid onto $1.4-1.8 \mathrm{ml}$ of bulk solution. They point out that the negative macromolecular concentration gradient at the leading site of the band must be compensated by a positive density gradient to prevent convection. This is done by providing a slightly higher density in the bulk solution, often using mixtures with $\mathrm{D}_{2} \mathrm{O}$ or solutions of concentrated electrolyte. Vinograd had a closer look at the method and investigated different centerpieces (Vinograd et al. 1965), checked the conditions of the experiment (Vinograd and Bruner 1966b) and investigated nucleic acids using ABC (Vinograd and Bruner 1966a). Lebowitz et al. performed the ABC experiment with very low salt concentrations (Lebowitz et al. 1998) and also applied it for the investigation of binding of promoter DNA to SmtB protein (Kar et al. 2001). All of these experiments were evaluated to yield the weight average sedimentation coefficient via the movement of the sedimenting boundary and not the sedimentation coefficient distribution.

The ABC experiment also offers the possibility of bringing two solutions together, which react with each other and characterize the resulting product in situ, as the overlay occurs instantaneously during acceleration. This is a special case in the application of the ABC experiment and was first used for the characterization of active enzymes (Cohen and Mire 1971b). By the overlay of an enzyme solution onto the substrate solution, the enzyme can be observed in its active state, even without purification. This is a nice example for the big advantage of ABC over SV that the observation of chemical reactions is possible and the application for active enzyme investigation was also repeated by others (Cohen and Claverie 1975; Cohen and Mire 1971a; Harding and Rowe 1996; Kemper and Everse 1973; Wolodko et al. 1986). Analysis was performed by a logarithmic plot of the position of the maxima versus time according to $\ln r_{0}=s \omega^{2} t+$ constant, which gives a weight average sedimentation coefficient.

Another example for application of $\mathrm{ABC}$ to perform a chemical reaction directly in the AUC cell is the so-called "Synthetic boundary crystallization" (Börger and Cölfen 1999; Börger et al. 2000). Here, a sodium sulfide solution is layered on top of a cadmium chloride solution containing thioglycerol as stabilizer. At the interface between the solutions, the solubility product is exceeded and CdS nanoparticles are formed. This way, nucleation species can be analyzed with an Angström resolution.

The same principle of performing a reaction inside the AUC was used in the investigation of silver nucleation species (Völkle et al. 2015). The overlay of a reducing agent onto 
a silver ion solution formed silver clusters, which were characterized with Angström size resolution as eight different, very small species. An overview of the ABC experiment and its applications is also given in literature (Schuck et al. 2016).

For the evaluation of SV AUC data, mainly two different groups of algorithms exist (Walter 2017): The parameter free approaches including $\mathrm{d} c / \mathrm{d} t$ (Stafford 1992), vanHoldeWeischet (vHW) analysis (Van Holde and Weischet 1978) and wide distribution analysis (WDA) (Stafford and Braswell 2004), and boundary fitting algorithms like Finite element modeling in ULTRASCAN (Demeler and Saber 1998), $1 s-g\left(s^{*}\right)$ and $c(s)$ in SEDFIT (Schuck 2000) and Lamm equation fitting in SEDANAL (Stafford and Sherwood 2004). For those, the experimental data is fitted to numerical solutions of the Lamm equation, which describes the sample concentration changes with time and location during an AUC Run. Generally, all these possibilities for analysis are offered by programs like SEDFIT (Schuck 2000), ULTRASCAN (Demeler 2005) and SEDANAL (Sherwood and Stafford 2016). There are a variety of possibilities for the analysis of SV data, but just very few methods for the evaluation of the $\mathrm{ABC}$ experiment. SEDFIT, ULTRASCAN and SEDANAL offer a possibility for the analysis of this experiment type with special implemented methods. In ULTRASCAN and SEDANAL, it is even possible to analyze multiwavelength (MWL) data obtained by the MWL-detector (Karabudak et al. 2016; Walter et al. 2015). However, all analysis methods do not yet consider the difference in density created by the overlay solution. In SEDFIT, the tool for analyzing ABC data is the "Analytical zone centrifugation". Here, the overlaying process is assumed to be ideal and instantaneous and the initial state is modeled as a step function. Especially for non-ideal samples with significant polydispersity, this results in high root mean squared deviation (RMSD) values for the Fits. In SEDANAL, Fits to the Lamm equation can be performed selecting the synthetic boundary experiment and choosing the first scan as initial scan. This tool is now implemented since version 6.92 (Correia et al. 2018). Overall, although there are a lot of powerful possibilities of analyses offered by modern programs, analyses of ABC experiments are still underdeveloped and do not profit from the variety of tools meanwhile accessible for SV experiments.

To improve this situation, we revisit ABC experiments to take a closer look into the analysis possibilities for this experiment and identify issues during analysis, which cause problems in comparison to the SV analysis.

\section{Materials and methods}

\section{Materials}

Myoglobin from horse skeletal muscle, 95-100\%, lyophilized powder, was purchased from Sigma (Sigma-Aldrich
Chemie GmbH, Riedstr. 2, D-89555 Steinheim, SigmaAldrich Co., 3050 Bruce Street, St Louis, MO 63103 USA) and used as received. Sodium chloride was purchased from Fluka (Fluka Chemie GmbH CH 9471 Buchs, Switzerland) and used as received. A nanoparticle size standard based on monodisperse polystyrene (PS) of $100 \mathrm{~nm}$ size was obtained from Sigma-Aldrich (Sigma-Aldrich AG, Industristrasse 25, CH 9471 Buchs, Switzerland) in aqueous suspension and used as received. For all dilutions, water of Milli-Q quality was used, sourced from a Milli-Q Synthesis A10 system equipped with a Quantum EX Ultrapure Organex cartridge (Merck Millipore, Am Kronenberger Hang 5, D-65824 Schwalbach, Germany). $\mathrm{D}_{2} \mathrm{O}$ was purchased from Aldrich (Sigma-Aldrich Chemie GmbH, Riedstr. 2, D-89555 Steinheim, Sigma-Aldrich Co., 3050 Bruce Street, St Louis, MO 63103 USA) and used as received.

\section{Instrument and experiment}

For all measurements, an UV/Vis multiwavelength AUC was used. The general setup is described in literature (Bhattacharyya et al. 2006; Strauss et al. 2008; Walter et al. 2014).

For both myoglobin and polystyrene size standard, SV and $\mathrm{ABC}$ experiments were conducted. For the SV experiments cells were equipped with a $12 \mathrm{~mm}$ titanium standard centerpiece. For the ABC experiment, a $12 \mathrm{~mm}$ charcoalfilled Epon Beckman ABC centerpiece of the Vinograd type was used (Vinograd et al. 1965). This provides a small $(15 \mu \mathrm{l})$ reservoir, which is connected with the sample sector via a thin capillary.

The measurements of myoglobin were performed at $25^{\circ} \mathrm{C}$ and a rotor speed of 60,000 RPM. For the SV run, the sample sector was filled with $340 \mu \mathrm{l} 0.5 \mathrm{mg} / \mathrm{ml}$ myoglobin solution in $150 \mathrm{mM} \mathrm{NaCl}$ in $\mathrm{D}_{2} \mathrm{O}$. The reference sector was filled with $360 \mu \mathrm{l}$ of $150 \mathrm{mM} \mathrm{NaCl}$ in $\mathrm{D}_{2} \mathrm{O}$. For the $\mathrm{ABC}$ experiment, the reservoir was filled with $1 \mu \mathrm{l}$ of $15 \mathrm{mg} / \mathrm{ml}$ myoglobin solution in $150 \mathrm{mM} \mathrm{NaCl}$ in $\mathrm{H}_{2} \mathrm{O}$. The sample sector was filled with $290 \mu \mathrm{l}$ of $150 \mathrm{mM} \mathrm{NaCl}$ in $\mathrm{D}_{2} \mathrm{O}$. The reference sector was filled with $330 \mu \mathrm{l}$ of $150 \mathrm{mM} \mathrm{NaCl}$ in $\mathrm{D}_{2} \mathrm{O}$.

The measurements of the polystyrene size standard were performed at $20{ }^{\circ} \mathrm{C}$ at $11000 \mathrm{RPM}$. For the SV run, $340 \mu \mathrm{l}$ of a dilution of 1:100 of PS standard suspension in $\mathrm{H}_{2} \mathrm{O}: \mathrm{D}_{2} \mathrm{O}=7: 3$ was filled in the sample sector. The reference sector was filled with $360 \mu \mathrm{H}_{2} \mathrm{O}$. For the ABC experiment, $1 \mu \mathrm{l}$ of a dilution of PS standard suspension 1:1 in $\mathrm{H}_{2} \mathrm{O}$ was filled into the reservoir. The sample sector was filled with $290 \mu \mathrm{l}$ of $\mathrm{H}_{2} \mathrm{O}: \mathrm{D}_{2} \mathrm{O}=7: 3$. The reference sector was filled with $330 \mu \mathrm{l}$ of $\mathrm{H}_{2} \mathrm{O}$. 


\section{Data processing and analysis}

The raw data were processed, integrated and derived for analysis by the system design software LabVIEW 2016 (32 bit) from National Instruments. Also, the moving boundary method was implemented into this software using $\ln r_{0}=s \omega^{2} t+$ constant.

For analysis, $c(s)$ and analytical zone centrifugation $c(s)$ from SEDFIT Version 15.01b (Lebowitz et al. 1998; Schuck 2000), vHW from ULTRASCAN Version 4.0 (Demeler and van Holde 2004) and the $\mathrm{d} c / \mathrm{d} t$ Analysis, WDA Analysis and Lamm equation fitting (for both SV and ABC/synthetic boundary experiments) from SEDANAL Version 6.92 (Stafford 1992; Stafford and Braswell 2004; Stafford and Sherwood 2004) were used. For the plots of data fitting and the residuals from the SEDFIT data, GUSSI was used (Brautigam 2015).

Analysis of the $\mathrm{ABC}$ experiments on myoglobin and PS latex in ULTRASCAN with the ABC analysis tool did not deliver satisfying results for the here applied model systems, resulting in modeled data which were off the original data with very high RMSD values. Therefore, this analysis tool could not be considered further here.

For the $\bar{v}$ of myoglobin, the value calculated from the sequence was used: $0.7468 \mathrm{ml} / \mathrm{g}$. For the solvent values for the $150 \mathrm{mM} \mathrm{NaCl}$ solution in $\mathrm{D}_{2} \mathrm{O}, 1.1045 \mathrm{~g} / \mathrm{ml}$ for the density and $1.095 \mathrm{cP}$ for the viscosity were used. The values for the density and viscosity were obtained using the density meter DMA $5000 \mathrm{M}$ and microviscometer Lovis 2000ME from Anton Paar (Anton Paar, Anton-Paar-Straße 20, AU-8054 Graz, Austria).

For the polystyrene, $0.9524 \mathrm{ml} / \mathrm{g}$ was used for the value of $\bar{v}$ and $1.0293 \mathrm{~g} / \mathrm{ml}$ for the density and $0.9522 \mathrm{cP}$ for the viscosity of the 7:3 mixture of $\mathrm{H}_{2} \mathrm{O}: \mathrm{D}_{2} \mathrm{O}$. The values for the density and viscosity were obtained plotting values for the pure components and linear extrapolation for the mixture.

\section{Results and discussion}

To address the problem of analysis of the data from $\mathrm{ABC}$ experiments by the common programs for the analysis of SV experiments, we integrated the ABC data to give sigmoidal curves as obtained in $\mathrm{SV}$ experiments as presented in Fig. 2c. We like to test the integration of $A B C$ data as a model-free approach for the analysis of $\mathrm{ABC}$ data. This avoids problems with the boundary conditions for the so far existing modeling approaches and could therefore improve the situation of $\mathrm{ABC}$ analysis. This is not based on simulations, modeling or theoretical calculations and does not take into account any assumptions. There are also no boundary conditions used in this approach. The investigation of various parameters on the ABC experiment, which can help the
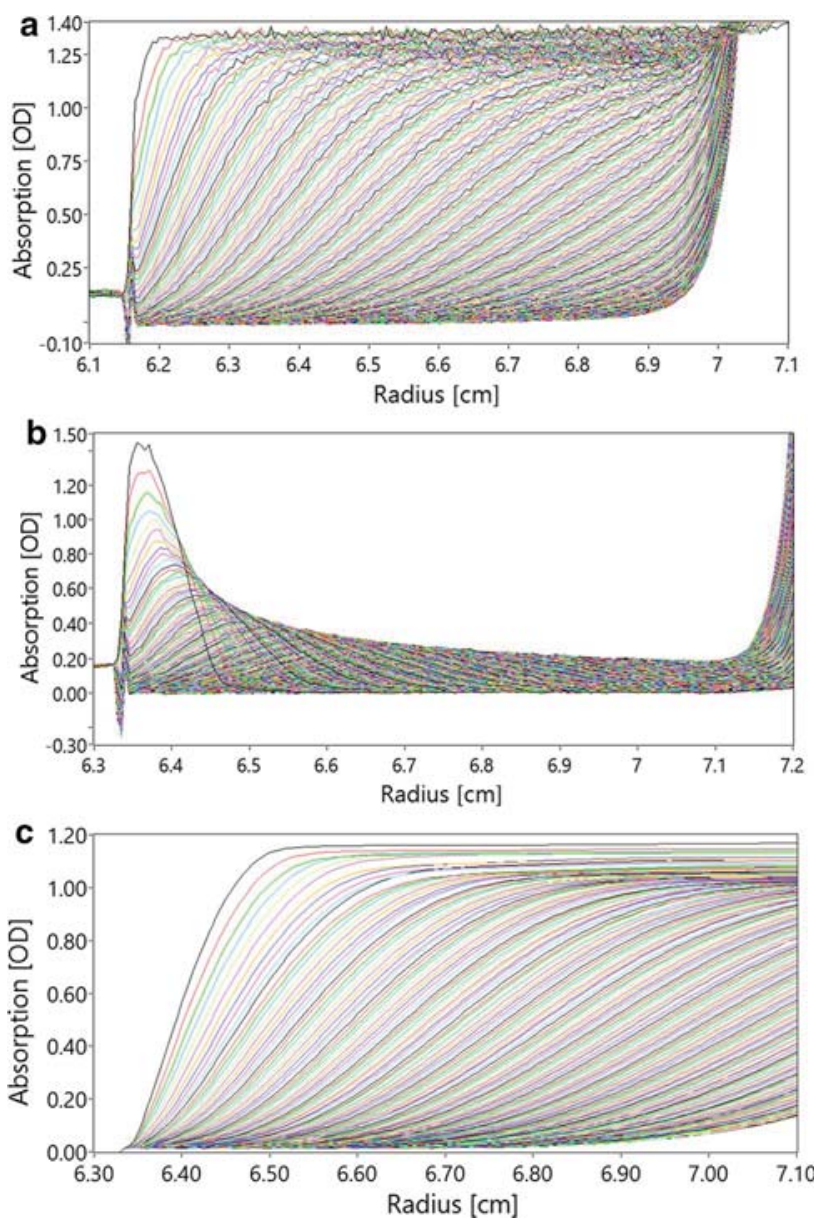

Fig. 2 Raw data of an AUC Run with myoglobin in $150 \mathrm{mM} \mathrm{NaCl}$ in $\mathrm{D}_{2} \mathrm{O}$ at $280 \mathrm{~nm}$. a SV experiment, b ABC experiment, c integrated data of the $\mathrm{ABC}$ experiment

definition of boundary conditions for modeling, are extensive and therefore are addressed in a separate study, which is unpublished yet. To test if these integrated data sets give the same results as the standard SV experiments, the wellcharacterized protein myoglobin was used as a test system. This protein provides a defined globular shape, both molecular weight and partial specific volume are known (Ehrenberg 1957; Oord et al. 1969) and is therefore suitable for performing test runs. Both SV and ABC experiments with myoglobin were performed using the same solvent. Naturally, for the $\mathrm{ABC}$ experiment, the myoglobin concentration was the 30 -fold of the concentration in the SV experiment, as also the overlay volume is $1 / 290$ of the volume in the sample sector. Figure 2 shows the comparison of the received raw data at $280 \mathrm{~nm}(\mathrm{a}: \mathrm{SV}, \mathrm{b}: \mathrm{ABC})$ and the data received by integration of the $\mathrm{ABC}$ experiment (c). For the integration, the baseline of Scan 1 was used as baseline. Integration started from Meniscus position. The quality of the obtained integrated data is comparatively good. The signal to noise ratio is $0.1 \%$, whereas the $\mathrm{SV}$ data exhibit a $s / n$ of $1.28 \%$. Noise 
is smoothed out with the integration of the data. Comparison of the data illustrates that the integrated $\mathrm{ABC}$ data seems to have a lower slope than data from SV. This indicates a term relevant in $\mathrm{ABC}$, which is not existent in the $\mathrm{SV}$ experiment.

First, analysis of the above experiments at $280 \mathrm{~nm}$ was performed with parameter free approaches as $\mathrm{d} c / \mathrm{d} t$ and WDA (Stafford 1992; Stafford and Braswell 2004), moving boundary method and vHW analysis (Demeler and van Holde 2004). The results are shown in Table 1. The corresponding analyses are shown in Figs. S2, S3 for the moving boundary, Figs. S6, S7 for the d $c / d t$ and Figs. S8, S9 for WDA analysis and Figs. S10, S11 for vHW analysis.

The sedimentation coefficient values from both SV and integrated $\mathrm{ABC}$ data are in very good agreement for the moving boundary analysis, which we take as reference here. Both show values around $1.3 \mathrm{~S}$ as expected for monomeric myoglobin in $\mathrm{D}_{2} \mathrm{O}$ at $25^{\circ} \mathrm{C}$, which gives $s_{20, \mathrm{w}}=2.06 \mathrm{~S}$ consistent with the $s_{20, \mathrm{w}}$ of $2 \mathrm{~S}$ reported in the literature (Ehrenberg 1957; Helwig and Greenberg 1952; Oord et al. 1969). For the $\mathrm{d} c / \mathrm{d} t$ method, a deviation in $s$ value around $0.25 \mathrm{~S}$ can be determined between the analyses of the two methods. The results for the WDA analysis also differ by $0.25 \mathrm{~S}$; both values seem to be shifted more to lower sedimentation coefficients compared to the other methods. This deviation

Table 1 Analysis of a myoglobin AUC dataset at $280 \mathrm{~nm}$ with sedimentation velocity and integrated $\mathrm{ABC}$ data using different methods

\begin{tabular}{lllll}
\hline Experiment type & $\mathrm{d} c / \mathrm{d} t(\mathrm{~S})$ & WDA $(\mathrm{S})$ & $\begin{array}{l}\text { Moving } \\
\text { boundary } \\
(\mathrm{S})\end{array}$ & $\mathrm{vHW}(\mathrm{S})$ \\
\hline Sedimentation velocity & 1.18 & 1.03 & 1.28 & 1.93 \\
Integrated ABC & 1.43 & 1.29 & 1.29 & 1.39 \\
\hline
\end{tabular}

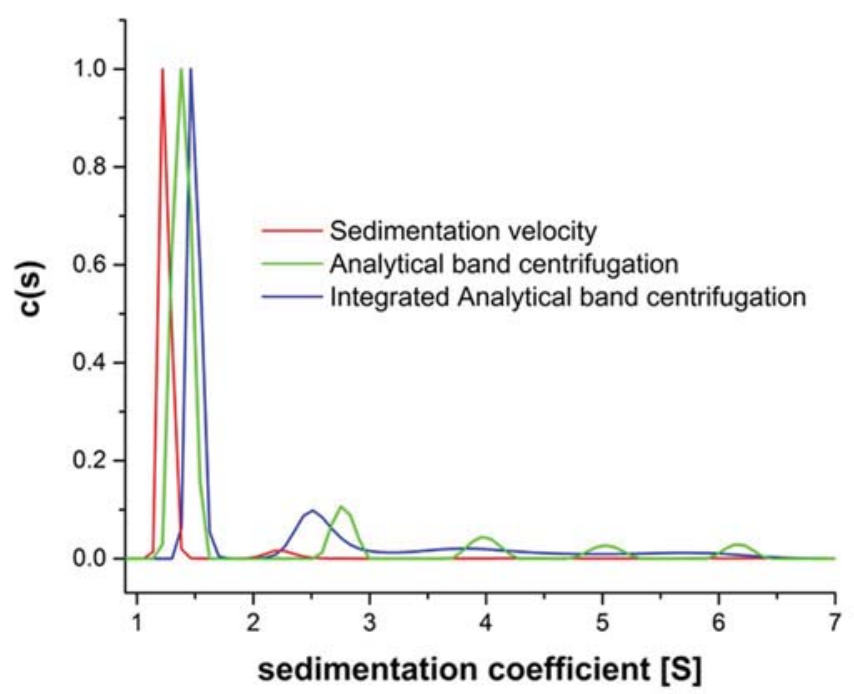

is even more pronounced for the vHW analysis with $0.54 \mathrm{~S}$. The analysis of the SV data delivers the significantly higher sedimentation coefficient. The validity of boundary modeling was tested using the $c(s)$ distribution in SEDFIT. The distributions of both SV and ABC experiment analysis via Analytical Zone centrifugation and analysis of the integrated $\mathrm{ABC}$ data, which are treated like SV data, are shown in Fig. 3. Both $c(s)$ distribution and converted $c(s)_{20, \mathrm{w}}$ are plotted. The corresponding fits are shown in Figs. S15, S16, and S17.

As mentioned above, the values from SV are typical for myoglobin. The transformation of the sedimentation coefficients in the experiment $(1.3 \mathrm{~S})$ to $s_{20, \mathrm{w}}$ values delivers a value of around $2.0 \mathrm{~S}$, which is exactly the value mentioned in literature (Ehrenberg 1957; Helwig and Greenberg 1952; Oord et al. 1969). Still, the distributions differ in between the different analysis methods. Generally, a shift to higher sedimentation coefficients (SV: $s=1.22 \mathrm{~S}, \mathrm{ABC}: s=1.38 \mathrm{~S}$; integrated $\mathrm{ABC}: s=1.46 \mathrm{~S}$ ) is visible in the ABC experiments, which is even more pronounced for the distribution of the integrated data, consistent with the $\mathrm{d} c / \mathrm{d} t$ results in Table 1. But, it is also obvious that the Analytical Zone centrifugation tool shows the same discrepancy, since the agreement between these data and the integrated $\mathrm{ABC}$ data is reasonable. This deviation is probably caused by differences between SV and ABC experiment, which are not considered in the analysis.

In Table 2, results received from performing Lamm equation fitting using SEDANAL are shown. The corresponding fits are shown in Figs. S12, S13, and S14. The same trend is visible for both boundary fitting methods. The sedimentation coefficients seem to be overestimated for the $\mathrm{ABC}$ and the integrated $\mathrm{ABC}$ analysis. This shows that both, the so far

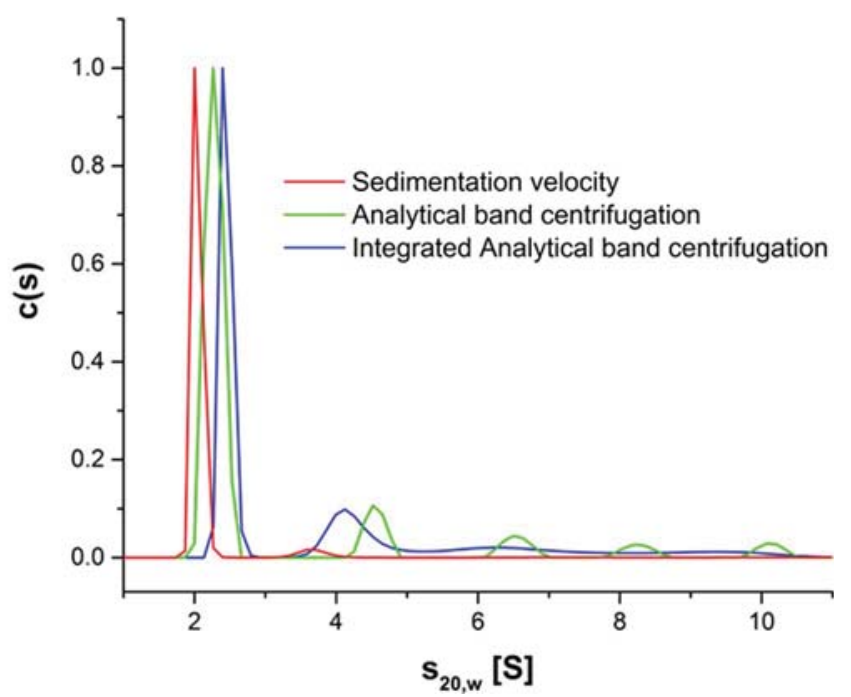

Fig. 3 Sedimentation coefficient distribution $c(s)$ and $c(s)_{20, \mathrm{w}}$ of SV, ABC and integrated ABC data of myoglobin at $280 \mathrm{~nm}$ 
existing analysis methods based on models (SEDFIT Analytical Zone centrifugation and the Lamm equation fitting in SEDANAL to synthetic boundary experiments) and our model-free approach using the integrated data set, show an offset to the reference of the SV dataset here. Still, the results received from these different methods are in a similar range and point towards another term, which seems to play a role in the $\mathrm{ABC}$ experiment, but not in the SV experiment and therefore leads to a shift of the obtained analysis values. In the ABC experiment, the boundary of overlaid concentrated sample is initially very thin, sharp and defined, but diffusion of the sample into the solvent already upon acceleration causes broadening and smearing of the boundary, so that the observed initial band is no step function, which it should be (see Fig. 1). This is not the case in a SV experiment, as the sample is evenly distributed over the whole sample sector at the beginning of an experiment and therefor, the initial conditions are precisely known. Broadening of the sedimenting boundary during the experiment by diffusion of the particles is considered in the common evaluation approaches. The broadening of the initial band after overlay with respect to the expected step function causes a difference, which affects the analysis of the integrated ABC data as well as the Analytical Zone analysis in SEDFIT. As myoglobin is very small, diffusion plays a significant role during the experiment. As we assume, this causes one of the key issues with

Table 2 Analysis using SEDANAL of a myoglobin AUC dataset at $280 \mathrm{~nm}$ of sedimentation velocity, $\mathrm{ABC}$ and integrated $\mathrm{ABC}$ data

\begin{tabular}{ll}
\hline Experiment type & Fit (S) \\
\hline Sedimentation velocity & 1.28 \\
ABC & 1.42 \\
Integrated ABC & 1.86 \\
\hline
\end{tabular}

the analysis, we conclude that this problem does not have as much impact when using nanoparticles with less diffusion. Therefore, we performed measurements with a polystyrene size standard of $100 \mathrm{~nm}$ size, with a diffusion coefficient, which is a factor of 22 smaller than that of myoglobin. These are very monodisperse polystyrene beads of known size and the diffusion broadening of their sedimentation boundaries is considered to be negligible. The evaluation of the integrated $\mathrm{ABC}$ data, the ABC data via the Analytical Zone centrifugation tool and of the $\mathrm{SV}$ data, all at $360 \mathrm{~nm}$, are shown in Fig. 4, corresponding Fits can be found in Figs. S18, S19, and S20. Also, the plot of the raw data (Fig. S1) and of the moving boundary evaluation (Figs. S4, S5) are shown in the supporting information.

The distribution of the PS Latex is generally broader than the distribution of the myoglobin data, which is also related to the existing slight polydispersity of the sample, which is natural for nanoparticles-even for calibration standards as the used one-especially in comparison to the monodisperse protein myoglobin, but the particle sizes of the peak maxima agree quite well at the value around $100 \mathrm{~nm}$ (SV: $d=100.2 \mathrm{~nm}, \mathrm{ABC}: d=103.9 \mathrm{~nm}$; integrated ABC: $d=105.1 \mathrm{~nm})$.

It is noticeable that the peak maxima of the sedimentation coefficient distributions exhibit the same shift to higher values for $\mathrm{ABC}$ experiments as the analyses of the myoglobin dataset. Also, the ABC particle size distributions from both evaluations agree reasonably but are by far broader, than the one from SV, which shows the expected narrow distribution for a particle size calibration standard. As the values for the different methods still show a pronounced discrepancy, this suggests that there is still another term in the ABC experiment, besides diffusion, which is not considered so far. This
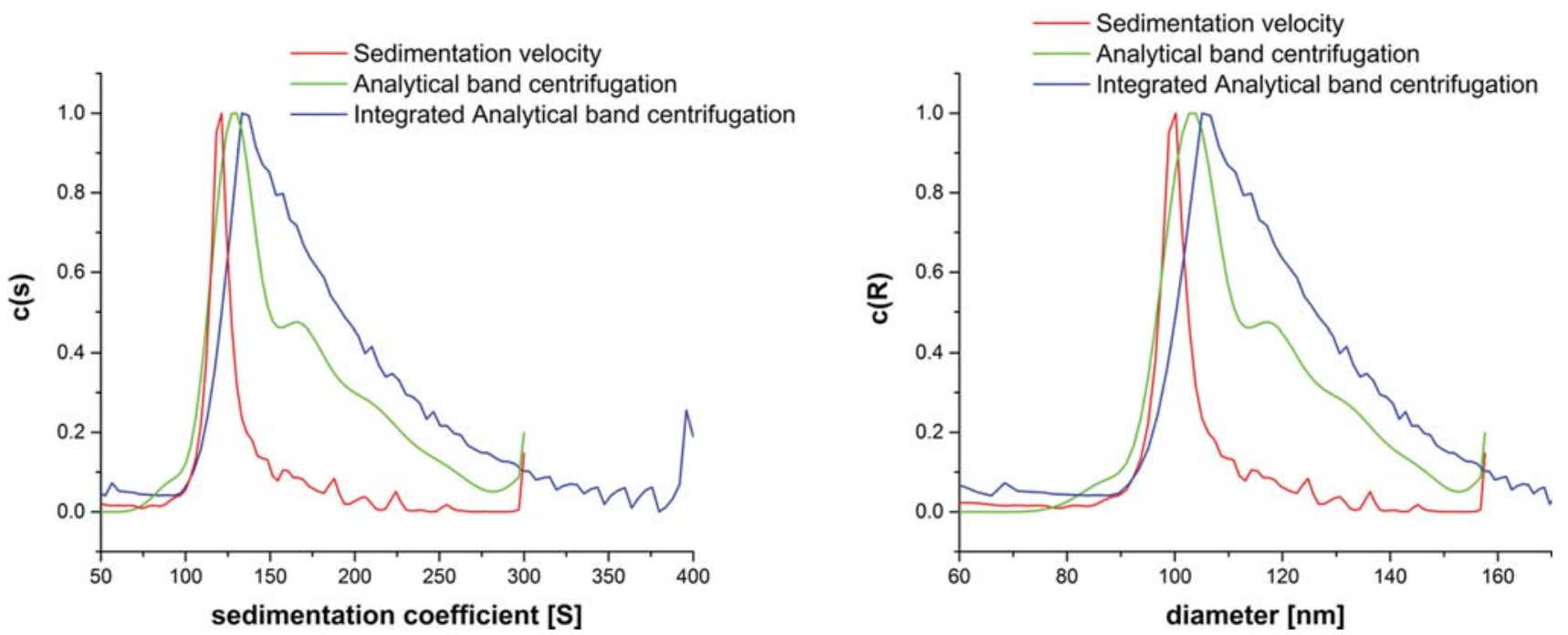

Fig. $4 c(s)$ and $c(R)$ distribution of $100 \mathrm{~nm}$ size standard polystyrene latex of $\mathrm{SV}, \mathrm{ABC}$ and integrated $\mathrm{ABC}$ experimental data at $360 \mathrm{~nm}$ 
could be the dynamic density gradient between the solvent $\mathrm{D}_{2} \mathrm{O}$ and the overlaid $\mathrm{H}_{2} \mathrm{O}$ from the reservoir, which is not considered by any analysis method. As these two solvents are diffusing into each other during the time of the experiment, this diffusion also causes broadening of the boundary, where the particles are present. This is not considered in the analysis and therefore cannot be fitted for and causes deviations, but it also cannot be prevented, as it is necessary for the stability of the forming band. The density gradient, which builds up in the cell can be calculated by the integrated form of Fick's second law for the boundary condition of a diffusion layer: $c(x, t)=\frac{n}{A \sqrt{\pi D t}} \exp \left(\frac{-x^{2}}{4 D t}\right)$, where $n$ is the amount of the diffusing component (in this case the overlaid water), $D$ the diffusion coefficient of $\mathrm{H}_{2} \mathrm{O}$ in $\mathrm{D}_{2} \mathrm{O}, A$ the interface area, $x$ the local position from start and $t$ the time.

With this formula, the development of the gradient over the time and the radius can be calculated (see Fig. 5). The diffusion coefficient of $\mathrm{H}_{2} \mathrm{O}$ in $\mathrm{D}_{2} \mathrm{O}$ of $6.27 \times 10^{-7} \mathrm{~cm}^{2} / \mathrm{s}$ can be determined via plotting of $\left(\frac{c_{\mathrm{P}}}{\mathrm{d} c / \mathrm{d} r}\right)^{2}$ versus $t$ and dividing the slope of the resulting line by $4 \pi$ (Ralston 1993). The plot is shown in Fig. S21. The values for $\left(\frac{c_{\mathrm{P}}}{\mathrm{d} c / \mathrm{d} r}\right)^{2}$ were determined from Interference scans taken during the overlay of $1 \mu \mathrm{l}$ water onto $\mathrm{D}_{2} \mathrm{O}$. A movie created from these scans is available in the supporting information, which shows the development of the density gradient over time. The calculation of the density gradient based on these values is shown in Fig. 5a for an overlay of $1 \mu \mathrm{H}_{2} \mathrm{O}$ onto $\mathrm{D}_{2} \mathrm{O}$. This gives quantitatively the amount of water at every cell position in every scan. This plot can be used for the calculation of the

\section{a}

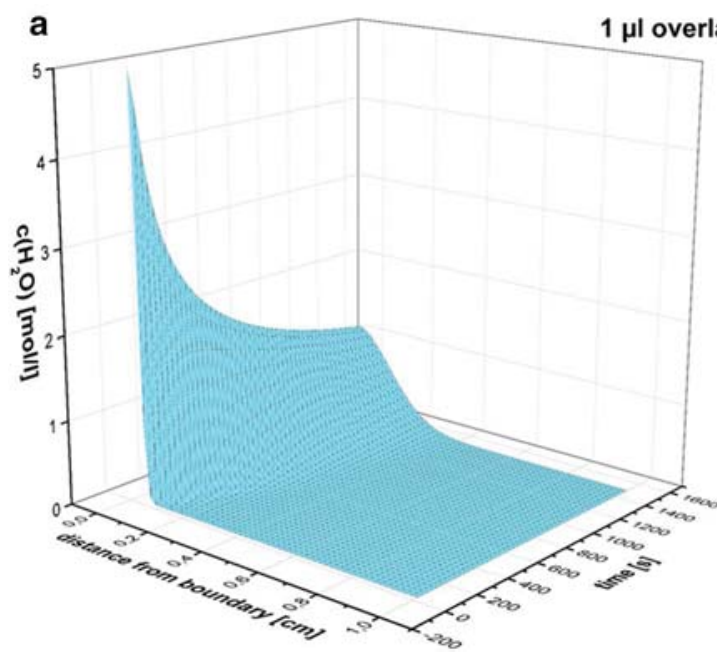

Fig. 5 Simulation of a dynamic density gradient between $\mathrm{H}_{2} \mathrm{O}$ and $\mathrm{D}_{2} \mathrm{O}$ with an overlay of $1 \mu 1 \mathrm{H}_{2} \mathrm{O}$ onto $\mathrm{D}_{2} \mathrm{O}$ using the integrated form of Fick's second law. Shown is the concentration of $\mathrm{H}_{2} \mathrm{O}$ versus time and the distance from the boundary in the cell (a). $1 \mathrm{~cm}$ distance cor- deviation of the $s_{20, \mathrm{w}}$ value by the error in density and viscosity caused by the overlaid and diffusing water. A linear concentration dependency of the density and viscosity of a mixture of $\mathrm{H}_{2} \mathrm{O}$ and $\mathrm{D}_{2} \mathrm{O}$ are assumed. The results of this calculation are shown in Fig. 5b. They show the quantitative influence of the error in s caused by the density gradient. The maximal effect of the density gradient is $6 \%$ at the maximum directly at the boundary immediately after the overlay. After $300 \mathrm{~s}$, the value has dropped under a deviation of $3 \%$ of the $s_{20, w}$ value, which is after the second scan for the myoglobin experiment. Therefore, exclusion of the first few scans from analysis is a sufficient treatment to maintain an error $<3 \%$ by a non-constant density and viscosity.

But, the shape of the initial boundary is deformed, as it passes through this ill-defined zone respective density and viscosity, which also explains the moderate to bad fitting performance of boundary fitting methods, which actually rely on the initial boundary being a step function (see Figs. S16 and S 19). Therefore, it is obvious that the density difference between the two solutions is as small as possible. Still, the question remains how slight the density difference can be to still provide a proper overlay. For the crystallization of CdS nanoparticles (Börger and Cölfen 1999; Börger et al. 2000), a density difference of only $0.006 \mathrm{~g} / \mathrm{ml}$ was adequate for an overlay, which is very remarkable. For the myoglobin experiment performed in this study, raw data were collected for different solvent densities and only for an overlay onto pure $\mathrm{D}_{2} \mathrm{O}$ a stable boundary was observed, as also shown in Fig. S22. This could be also related to the higher density and viscosity caused by the high concentration of myoglobin $(15 \mathrm{mg} / \mathrm{ml})$. This solution has a density of $1.002 \mathrm{~g} / \mathrm{ml}$ and therefore, the formation of a good band

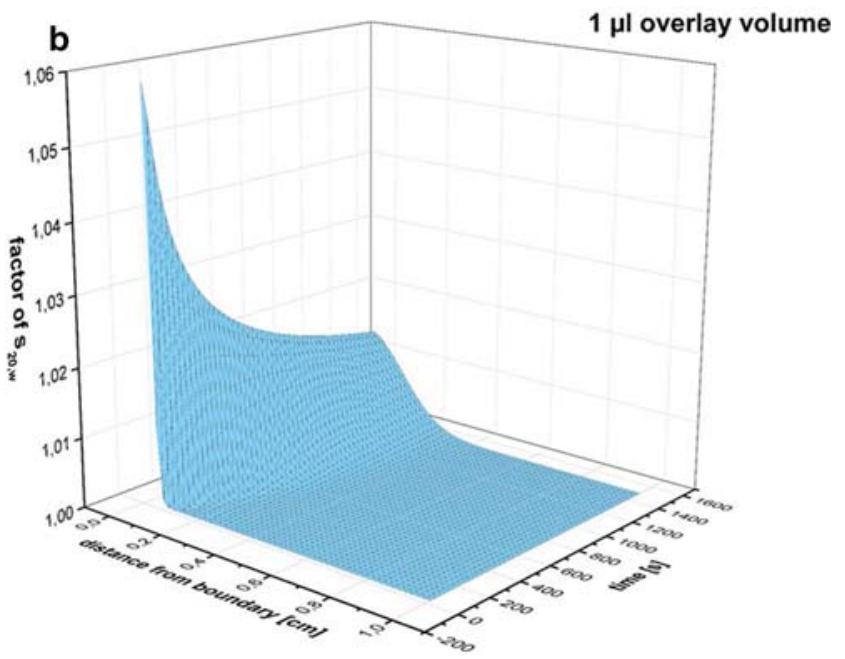

relates to the cell bottom at $7.1 \mathrm{~cm}$. In (b), the corresponding deviation of the $s_{20, \mathrm{w}}$ value by the error in density and viscosity due to the overlaid and diffusing water is plotted versus time and distance to the boundary 
could have been expected already for a 1:1 mixture between $\mathrm{H}_{2} \mathrm{O}$ and $\mathrm{D}_{2} \mathrm{O}$. Obviously, it is sample dependent how large the density difference between solvent and overlaid solution can be to form a stable band.

\section{Conclusion}

Our results show that the idea of integrating data obtained from ABC experiments and analyzing them with the standard SV still needs some adjustments, but is applicable at least for parameter free approaches as the moving boundary method and eventually $\mathrm{d} c / \mathrm{d} t$ and gives comparable results to the existing $\mathrm{ABC}$ modeling tools. The problem is the necessary density difference between solvent and overlaid solution to form a sharp band. This leads to a dynamic density gradient. Its influence can be precisely determined via the integral form of Fick's second law for different overlay volumes and also its effect on the error in $s_{20, \mathrm{w}}$. Therefore, it can potentially be implemented into the powerful boundary modeling techniques. Nevertheless, an additional still not yet considered problem is the deformation of the initial boundary by the movement of the sample band through the dynamic density gradient. Since the densities and viscosities of the dynamic density gradient at every position and time are known, it appears possible to consider the deformation of the sample band by the dynamic density gradient in the evaluation of the data to be able to obtain true sedimentation coefficient distributions.

However, up to now, the different densities of the two solutions are not yet considered in any $\mathrm{ABC}$ evaluation approach. A potential workaround could be to define the first scan as initial boundary condition as is described in the SEDFIT manual (Schuck, http://www.analyticalultracentr ifugation.com/sedfit_help_analytical_zone_centrifugation. $\mathrm{htm}$ ) and in SEDANAL (Correia et al. 2018). But, this analysis would only be possible for a single species model, which would considerably limit the potential of $\mathrm{ABC}$, since it could not be used for any polydisperse or multi-species system. In addition, Fig. 5 shows that the dynamic density gradient is still effective after the first scan, which makes the first scan as initial boundary condition wrong if the density gradient is not considered.

Therefore, it seems very reasonable if the calculation of the varying solvent concentrations and viscosities in time and space by the integrated form of Fick's second law could be added to already existing tools for $\mathrm{ABC}$ evaluation like ULTRASCAN or SEDFIT to make ABC experiments available for the powerful existing analysis programs.

To conclude the results obtained in this work, the integration of raw data received from $\mathrm{ABC}$ experiments in the AUC potentially opens the analysis of these data to all modern tools of SV Analysis. Nevertheless, to decrease effects which are not considered in the SV analysis, it is recommended to overlay very small volumes $(1 \mu \mathrm{l})$ in the experiment and to keep the density difference as low as possible, which minimizes the effect of diffusion of the sample and the density gradient. Nevertheless, for a correct analysis of sedimentation coefficient distributions, the effect of the dynamic density gradient needs to be taken into account.

Acknowledgements We acknowledge funding of the Deutsche Forschungsgemeinschaft (DFG) within the NSF-DFG Materials World Network (GE 22278/6-1; CO 194/12-1).

\section{Compliance with ethical standards}

Conflict of interest The authors declare that they have no conflict of interest.

\section{References}

Bhattacharyya S, Maciejewska P, Börger L, Stadler M, Gülsün A, Cicek H, Cölfen H (2006) Development of a fast fiber based UVVis multiwavelength detector for an ultracentrifuge. In: Wandrey C, Cölfen H (eds) Analytical ultracentrifugation VIII vol 131 progress in colloid and polymer science. Springer, Berlin, pp 9-22. https://doi.org/10.1007/2882_002

Börger L, Cölfen H (1999) Investigation of the efficiencies of stabilizers for nanoparticles by synthetic boundary crystallization ultracentrifugation. Prog Colloid Polym Sci 113:23-28

Börger L, Cölfen H, Antonietti M (2000) Synthetic boundary crystallization ultracentrifugation: a new method for the observation of nucleation and growth of inorganic colloids and the determination of stabilizer efficiencies. Colloids Surf A 163:29-38

Brautigam CA (2015) Chapter five-calculations and publication-quality illustrations for analytical ultracentrifugation data. In: Cole JL (ed) Methods in enzymology, vol 562. Academic Press, New York, pp 109-133. https://doi.org/10.1016/bs.mie.2015.05.001

Cohen R, Claverie J-M (1975) Sedimentation of generalized systems of interacting particles. II. Active enzyme centrifugation-theory and extensions of its validity range. Biopolymers 14:1701-1716. https://doi.org/10.1002/bip.1975.360140812

Cohen R, Mire M (1971a) Analytical-band centrifugation of an active enzyme substrate complex. Eur J Biochem 23:276-281. https:// doi.org/10.1111/j.1432-1033.1971.tb01619.x

Cohen R, Mire M (1971b) Analytical-band centrifugation of an active enzyme-substrate complex. FEBS J 23:267-275

Cölfen H, Pauck T (1997) Determination of particle size distributions with Angström resolution. Colloid Polym Sci 275:175-180. https ://doi.org/10.1007/s003960050068

Correia JJ, Wright RT, Hayes D, Sherwood PJ, Stafford WF (2018) AUC measurements of diffusion coefficients of monoclonal antibodies in the presence of human serum proteins. Biophys $\mathbf{J}$ 114:62a. https://doi.org/10.1016/j.bpj.2017.11.387

Demeler B (2005) UltraScan-a comprehensive data analysis software package for analytical ultracentrifugation experiments. In: Scott DJ, Harding SE, Rowe AJ (eds) Analytical ultracentrifugation: techniques and methods. The Royal Society of Chemistry, Cambridge, pp 210-230. https://doi.org/10.1039/9781847552 617-00210

Demeler B, Saber H (1998) Determination of molecular parameters by fitting sedimentation data to finite-element solutions of the 
Lamm equation. Biophys J 74:444-454. https://doi.org/10.1016/ S0006-3495(98)77802-6

Demeler B, van Holde KE (2004) Sedimentation velocity analysis of highly heterogeneous systems. Anal Biochem 335:279-288

Ehrenberg A (1957) Determination of molecular weights and diffusion coefficients in the ultracentrifuge. Acta Chem Scand 11:1257-1270

Harding S, Rowe A (1996) Active enzyme centrifugation. In: Engel PC (ed) Enzymology LabFax. Bios Scientific, Oxford, pp 66-75

Helwig HL, Greenberg DM (1952) Preparation of purified myoglobin from skeletal muscle of the guinea pig. J Biol Chem 198:695-701

Kar SR, Lebowitz J, Blume S, Taylor KB, Hall LM (2001) SmtBDNA and protein-protein interactions in the formation of the cyanobacterial metallothionein repression complex: $\mathrm{Zn}^{2+}$ does not dissociate the protein-DNA complex in vitro. Biochemistry 40:13378-13389. https://doi.org/10.1021/bi011289f

Karabudak E et al (2016) Simultaneous identification of spectral properties and sizes of multiple particles in solution with subnanometer resolution. Angew Chem Int Ed 55:11770-11774. https://doi. org/10.1002/anie.201603844

Kemper DL, Everse J (1973) Active enzyme centrifugation. Methods Enzymol 27:67-82

Lebowitz J, Teale M, Schuck PW (1998) Analytical band centrifugation of proteins and protein complexes. Biochem Soc Trans 26:745-749. https://doi.org/10.1042/bst0260745

Mächtle W, Börger L (2006) Analytical ultracentrifugation of polymers and nanoparticles. Springer, Berlin

Oord AHA, Wesdorp JJ, Dam AF, Verheij JA (1969) Occurrence and nature of equine and bovine myoglobin dimers. FEBS J 10:140-145

Ralston G (1993) Introduction to analytical ultracentrifugation, vol 1. Beckman, California

Schuck P. http://www.analyticalultracentrifugation.com/sedfit_help_ analytical_zone_centrifugation.htm. Accessed 4 Feb 2018

Schuck P (2000) Size-distribution analysis of macromolecules by sedimentation velocity ultracentrifugation and Lamm equation modeling. Biophys J 78:1606-1619. https://doi.org/10.1016/S0006 -3495(00)76713-0

Schuck P, Zhao H, Brautigam CA, Ghirlando R (2016) Basic principles of analytical ultracentrifugation. CRC Press, Boca Raton

Sherwood PJ, Stafford WF (2016) SEDANAL: model-dependent and model-independent analysis of sedimentation data. In: Analytical ultracentrifugation. Springer, Berlin, pp 81-102

Stafford WF (1992) Boundary analysis in sedimentation transport experiments: a procedure for obtaining sedimentation coefficient distributions using the time derivative of the concentration profile. Anal Biochem 203:295-301. https://doi.org/10.1016/00032697(92)90316-Y

Stafford WF, Braswell EH (2004) Sedimentation velocity, multi-speed method for analyzing polydisperse solutions. Biophys Chem 108:273-279
Stafford WF, Sherwood PJ (2004) Analysis of heterologous interacting systems by sedimentation velocity: curve fitting algorithms for estimation of sedimentation coefficients, equilibrium and kinetic constants. Biophys Chem 108:231-243. https://doi.org/10.1016/j. bpc.2003.10.028

Strauss H, Karabudak E, Bhattacharyya S, Kretzschmar A, Wohlleben W, Cölfen H (2008) Performance of a fast fiber based UV/ Vis multiwavelength detector for the analytical ultracentrifuge. Colloid Polym Sci 286:121-128. https://doi.org/10.1007/s0039 6-007-1815-5

Van Holde KE, Weischet WO (1978) Boundary analysis of sedimentation-velocity experiments with monodisperse and paucidisperse solutes. Biopolymers 17:1387-1403. https://doi.org/10.1002/ bip. 1978.360170602

Vinograd J, Bruner R (1966a) Band centrifugation of macromolecules in self-generating density gradients. II. Sedimentation and diffusion of macromolecules in bands. Biopolymers 4:131-156

Vinograd J, Bruner R (1966b) Band centrifugation of macromolecules in self-generating density gradients. III. Conditions for convection-free band sedimentation. Biopolymers 4:157-170

Vinograd J, Bruner R, Kent R, Weigle J (1963) Band-centrifugation of macromolecules and viruses in self-generating density gradients. Proc Natl Acad Sci 49:902-910

Vinograd J, Radloff R, Bruner R (1965) Band-forming centerpieces for the analytical ultracentrifuge. Biopolymers 3:481-489. https ://doi.org/10.1002/bip.1965.360030409

Völkle C, Gebauer D, Cölfen H (2015) FD nucleation: high-resolution insights into the early stages of silver nucleation and growth. Faraday Discuss 179:59-77. https://doi.org/10.1039/C4FD00269E

Walter J (2017) Multidimensional characterization of nanoparticles by means of analytical ultracentrifugation. PhD thesis, FriedrichAlexander Universität Erlangen-Nürnberg

Walter J et al (2014) Multidimensional analysis of nanoparticles with highly disperse properties using multiwavelength analytical ultracentrifugation. ACS Nano 8:8871-8886. https://doi.org/10.1021/ nn503205k

Walter J, Sherwood PJ, Lin W, Segets D, Stafford WF, Peukert W (2015) Simultaneous analysis of hydrodynamic and optical properties using analytical ultracentrifugation equipped with multiwavelength detection. Anal Chem 87:3396-3403. https://doi. org/10.1021/ac504649c

Wolodko WT, Kay CM, Bridger WA (1986) Active enzyme sedimentation, sedimentation velocity, and sedimentation equilibrium studies of succinyl-CoA synthetases of porcine heart and Escherichia coli. Biochemistry 25:5420-5425. https://doi.org/10.1021/bi003 $67 \mathrm{a} 012$ 